\title{
Editorial
}

\section{Key Role of the Rational Laboratory Strategy in Diagnostic, Analytical and Forensic Toxicology}

\author{
Antonio Colatutto*
}

Department of Laboratory Medicine, Clinical Pathology, Hospital S. Maria della Misericordia, Udine

As in a Medicine it is hard task to define correctly the term poison, it is otherwise difficult to understand the accurate borders of the Toxicology as discipline itself.

Various sub-disciplines of Toxicology have been illustrated as Environmental Toxicology, Analytical Toxicology, Clinical Toxicology, Occupational Toxicology and Forensic Toxicology, and each one has its own peculiarity.

It is nevertheless not easy to define the borders between Pharmacology and Toxicology too, because the side effects of commonly used therapies could be extremely toxic, the effects of the Drug of Abuse are also toxic, and toxic substances could be found in soil, water and air. It is well known that Medicine is "rapidly changing in rapidly changing world", but the paramount progress in laboratory technology has positively influenced the whole Medicine diagnostic process and practice.

For these reasons a rational diagnostic strategy is mandatory to optimize the resources. The aim of the toxicological analysis is firstly to detect the toxicant, and to fulfill this scope so that the toxicologist can take advantage of improved technology. Even if new screening methods are easy to perform, highly automated and fit not only for highly specialized laboratory staff, confirmation analysis needs more sophisticated and extremely expensive technology. It is well known indeed that confirmation tests should be performed only by chromatographic technology, both gas chromatographic and liquid chromatographic coupled with mass spectrometry, and these kinds of tests need highly specialized personnel.

\section{DIFFERENT AIMS: CLINICAL/ANALYTICAL TOXICOLOGY VERSUS FORENSIC TOXICOLOGY}

It should be kept in mind that the main known difference between the Clinical/Analytical Toxicology and the Forensic Toxicology is the aim of the analysis. If the main target of Clinical toxicology is to detect the toxicant for the correct diagnosis and therapy, so as to help the clinician, Forensic analysis is not only involved in the detection of the toxicant itself, but also into the detection of the exact amount of toxicant and its relationship with supposed lethal dose and Justice.

\footnotetext{
*Address correspondence to this author at the Department of Laboratory Medicine/Clinical Pathology, Hospital S. Maria della Misericordia, Udine; Tel: 00390432 552406; Fax: 00390432552402

E-mail: colatutto.antonio@aoud.sanita.fvg.it
}

In Analytical Toxicology the limiting step is the "time", while in Forensic toxicology it is crucial to achieve incontestable laboratory data, and in this setting it is also crucial to define properly what Quality of Toxicological results [1-4].

Every analytical laboratory must comply with a standard set of procedures: Standard Operating Procedures, Procedural Manuals, Analytical Methods Files, Analytical Instrument Calibration, Quality Assurance Procedures and Quality Control Procedures. These items are planned as to standardize the quality of analysis and to enhance the proficiency and the efficiency of the system itself, and it is mandatory to emphasize that the laboratory team must follow these Guide Lines.

It could be a paradox but undoubtedly the paramount progress in laboratory instrumentation has led to an increased difficulty in data interpretation, and the reason of this is the increased sensitivity and sensibility: the improved and more specific and sensible Limits of Quantification (LOQ) and Limits of Detection (LOD) could also increase the complexity itself of the data interpretation as whole. Every method used must be validated, and method validation in Forensic Toxicology is a cornerstone in the whole analytical process.

In Method validation in Forensic Toxicology, Mario Zilli gives an account of relevance and reliability of results, both in analytical and forensic toxicology. In this paper Mario Zilli gives a neat explanation of "not ever friendly" sides of analytical toxicology as Uncertainty Calculation, Uncertainty of Repeatability Calculation and Uncertainty of Calibration Calculation.

In Emerging Biomarkers of Alcohol Consumption: Clinical and Forensic Applications, Vincenza Bianchi deals with alcohol addiction and alcohol related analytical problems. In this item it is possible to find a correct way to interpret critically the approach to the diagnosis of chronic alcohol abuse, to understand the reliability of "new" markers of alcohol abuse as Carbohydrate Deficient Transferrin (CDT), Ethylglucuronide (EtG) and Ethylsulfate (EtS), Fatty Acid Ethylesters (FAEE) and Phosphatidylethanol (PEth). Vincenza Bianchi also gives a thorough explanation of the sophisticated techniques, as Liquid Chromatography coupled to Mass Spectrometry, which allow the quantification of the afore mentioned analytes.

The Residential Treatment as a Rehabilitation Opportunity for Alcoholism Care, dealt by Giorgio Cerizza and Paola Ranalletti, is a comprehensive work in a field of 
increasing relevance. It should be kept in mind that toxicology does not relate only to pharmacological therapeutics, but it is the first step to properly diagnose a disease and to start treating the patient properly, so the close relation between the toxicologist and the psychiatrist is of extreme importance. Even if our mind is strictly turned to toxicological analysis the importance of listening and observing as a cornerstone in diagnostic workup could not be understood.

Lorenzo Desinan, Antonio Colatutto and Pierguido Sala in "The relevance of synergy between forensic pathologist and toxicologist in medico-legal autopsies" summarize their experience in the research on post mortem drug distribution both in conventional and alternative matrices. The study deals with the research and quantification of common and uncommon toxicants and drugs in different matrices in post mortem. [5-7] It should be kept in mind that usual pharmacokinetic and pharmacodynamics data like distribution volume, protein binding scarcely have to be taken into account in post mortem toxicology. The medical examiner and the forensic toxicologist should be aware that Clinical Pharmacology assumptions could be largely invalid in post mortem, because a lot of conditions as tissue distribution of xenobiotics, post mortem changes (i.e. putrefaction) and $\mathrm{pH}$ variations can affect most sophisticated results too [2-5]. The relationship between concentrations measured before and after death challenges the importance of quantitative analyses, for these reasons any deduction from post mortem concentration should be critically checked. If the risk of false positive or negative test is a real "nightmare" in common clinical practice, this possibility is even worse in forensic toxicology. For the Forensic Toxicologist the only realistic and possible solution is to interpret the data in synergy with the Forensic Pathologist, as to understand analytical data along with medical history and circumstantial data.

\section{REFERENCES}

[1] Flanagan RJ, Connally G. Interpretation of analytical toxicology results in life and post-mortem. Toxicol Rev 2005; 24: 51-6.

[2] Leikin J, Watson W. Post-mortem toxicology: what the dead can and cannot tell us. J Clin Toxicol 2003; 41: 47-56.

[3] Richardson T. Pitfalls in forensic toxicology. Ann Clin Biochem 2000; 37: 20-44.

[4] Yarema MC, Becker CE. Key concepts in post-mortem drug redistribution. Clin Toxicol 2005; 43: 235-41.

[5] Harding-Pick D, Fryc O. Assessing death by poisoning: does the medical history help? Med Sci Law 1991; 31:69-75.

[6] Prouty RW, Anderson WH. The forensic science implications of site and temporal influences on post-mortem blood drug concentrations. J Forensic Sci 1990; 35:243-70.

[7] Jetter W. Postmortem biochemical changes. J Forensic Sci 1959; 4: $330-41$.

(C) Antonio Colatutto; Licensee Bentham Open.

This is an open access article licensed under the terms of the Creative Commons Attribution Non-Commercial License (http://creativecommons.org/licenses/by$\mathrm{nc} / 3.0 /$ ), which permits unrestricted, non-commercial use, distribution and reproduction in any medium, provided the work is properly cited. 